OPEN ACCESS

Edited by:

Chella Kamarajan,

SUNY Downstate Medical Center.

United States

Reviewed by:

Valerie Cardenas,

Neurobehavioral Research

United States

Zhen Yuan,

University of Macau, China

*Correspondence:

Manli Huang

huangmanli@zju.edu.cn

Dongrong Xu

xu.dongrong@columbia.edu

Received: 18 August 2018 Accepted: 25 October 2018

Published: 05 December 2018

Citation:

Zhao Z, Li X, Feng G, Shen Z, Li S, Xu Y, Huang M and Xu D (2018) Altered Effective Connectivity in the Default Network of the Brains of First-Episode, Drug-Naive Schizophrenia Patients With Auditory Verbal Hallucinations.

Front. Hum. Neurosci. 12:456 doi: 10.3389/fnhum.2018.00456

\section{Altered Effective Connectivity in the Default Network of the Brains of First-Episode, Drug-Naïve Schizophrenia Patients With Auditory Verbal Hallucinations}

\author{
Zhiyong Zhao 1,2, Xuzhou Li ${ }^{3}$, Guoxun Feng ${ }^{4}$, Zhe Shen ${ }^{4}$, Shangda $\mathrm{Li}^{4}$, Yi Xu${ }^{5}$, \\ Manli Huang ${ }^{5 *}$ and Dongrong $X^{2 *}$ \\ ${ }^{1}$ Shanghai Key Laboratory of Magnetic Resonance, East China Normal University, Shanghai, China, ${ }^{2}$ New York State \\ Psychiatric Institute, Columbia University, New York, NY, United States, ${ }^{3}$ Key Laboratory of Brain Functional Genomics (MOE \\ and STCSM), Institute of Cognitive Neuroscience, School of Psychology and Cognitive Science, East China Normal \\ University, Shanghai, China, ${ }^{4}$ College of Medicine, Zhejiang University, Hangzhou, China, ${ }^{5}$ Department of Psychiatry, \\ The First Affiliated Hospital, Zhejiang University School of Medicine, The Key Laboratory of Mental Disorder's Management \\ of Zhejiang Province, Hangzhou, China
}

Although the default mode network (DMN) is known to be abnormal in schizophrenia (SZ) patients with auditory verbal hallucinations (AVHs), it is still unclear whether AVHs that occur in SZ are associated with certain information flow in the DMN. This study collected resting-state functional magnetic resonance imaging data from 28 first-episode, drug-naïve SZ patients with AVHs, $20 \mathrm{SZ}$ patients without AVHs, and 38 healthy controls. We used Granger causality analysis (GCA) to examine effective connectivity (EC) of two hub regions [posterior cingulate cortex (PCC) and anteromedial prefrontal cortex (aMPFC)] within the DMN. We used two-sample $t$-tests to compare the difference in EC between the two patient groups, and used Spearman correlation analysis to characterize the relationship between imaging findings and clinical assessments. The GCA revealed that, compared with the non-AVHs group, EC decreased from aMPFC to left inferior temporal gyrus (ITG) and from PCC to left cerebellum posterior lobe, ITG, and right middle frontal gyrus in SZ patients with AVHs. We also found significant correlations between clinical assessments and mean strengths of connectivity from aMPFC to left ITG and from PCC to left ITG. Moreover, receiver operating characteristic analysis revealed that the above-mentioned effective connectivities had a diagnostic value for distinguishing SZ patients with AVHs from nonAVHs patients. These findings suggest that AVHs in SZ patients may be associated with the aberrant information flows of the DMN, and the left ITG may probably serve as a potential biomarker for the neural mechanisms underlying AVHs in SZ patients.

Keywords: schizophrenia, auditory verbal hallucinations, resting-state functional magnetic resonance imaging, default mode network, Granger causality analysis, effective connectivity 


\section{INTRODUCTION}

Auditory verbal hallucinations (AVHs), a symptom that appears to be very pragmatic and is sensitive to the perceptions of sound that occur without corresponding external stimuli, have been demonstrated to exist in $60-90 \%$ of schizophrenia (SZ) cases (Alderson-Day et al., 2016). Several studies have examined brain activation in SZ patients with AVHs or hallucination predisposition (Allen et al., 2012; Alderson-Day et al., 2015, 2016). However, the neural basis of this phenomenon is still not well-understood so far.

Resting-state functional magnetic resonance imaging (rsfMRI) is a task-independent and non-invasive method to assess brain regional and neural circuitry functions (Biswal et al., 1995). According to fMRI findings in the literature, altered resting-state networks (RSNs) have been proposed to involve the psychopathological substrates of SZ (Alderson-Day et al., 2015, 2016; Cui et al., 2017). Default mode network (DMN), one of the most well-known RSNs, seems to play a prominent role in SZ patients (Menon, 2011; Woodward et al., 2011). This largescale brain network comprises anteromedial prefrontal cortex (aMPFC), posterior cingulate cortex (PCC), and medial temporal lobe including the hippocampus and the lateral temporoparietal area (Buckner et al., 2008). DMN related dysfunction has been reported to be one of the pathomechanisms associated with AVHs in SZ patients (Bastos-Leite et al., 2014; Alderson-Day et al., 2015, 2016). A recent study demonstrated that spatial and temporal instabilities in the DMN were correlated with the severity of hallucinations (Jardri et al., 2013). Another study examined the functional connectivity of two hub regions (PCC and aMPFC) within the DMN and found cross-network abnormalities between DMN and salience system in SZ patients with resistant AVHs compared with those without AVHs (Alonso-Solís et al., 2015). However, findings of changes in the DMN in SZ patients with AVHs were not consistent in the previous reports. Some studies demonstrated increased activations or connectivity in the DMN in SZ patients with hallucinations (Alderson-Day et al., 2015, 2016), whereas other studies found a decrease or no changes in the connectivity (Vercammen et al., 2010; Wolf et al., 2011). Therefore, the role of the DMN in AVHs of SZ patients still remains unclear. Although the variety of results might be related to many factors such as patient characteristics or methodological approaches employed in the analyses.

Effective connectivity (EC) is defined as the influence of one neuronal system on another (Zhao et al., 2016) and is increasingly recognized. Two approaches for studying EC, dynamic causal modeling (DCM) and structural equation modeling (SEM), have been commonly used in SZ patients. For instance, by using DCM, investigators found abnormal EC between anterior cingulate cortex (ACC) and cognition- and emotion-related regions in SZ patients compared with healthy control subjects (HCs) (Cui et al., 2015). In addition, disrupted information flows between thalamus, hippocampus, and auditory cortex were found in SZ patients with AVHs but not in those without AVHs (Li et al., 2017). Using the SEM method, Schlösser et al. (2003) revealed an abnormal pattern of connectivity in the cortical-cerebellar circuit in the SZ patient group relative to the HCs. Moran et al. (2013) identified a diminished positive outflow from dorsal anterior insula to central executive network and DMN in SZ using SEM. However, these models require assumptions about the existence of influence and its directionality between two regions, in which the directionality of the influence defines causality or dependency between the two regions. Therefore, any misspecification of the models may result in erroneous conclusions (Roebroeck et al., 2005; Sathian et al., 2011; Hutcheson et al., 2015). Granger causality analysis (GCA), an EC method that originated from the field of economics, can be used to describe observed data in terms of directed functional interactions or information flow (Zhao et al., 2017). Additionally, the GCA provides information about dynamics and directionality of fMRI BOLD signal in cortical circuits (Liao et al., 2011), which effectively compensates for the shortcomings of the two aforementioned approaches, as no prior knowledge is required (Roebroeck et al., 2005). This method has been employed to detect abnormal EC in SZ patients (Palaniyappan et al., 2013; de la Iglesia-Vaya et al., 2014; Guo et al., 2015). However, the GCA is rarely used to investigate AVHs-related EC changes in first-episode, drug-naïve SZ patients.

In this study, we first used the GCA to detect EC alterations of the DMN in SZ patients with AVHs versus those without AVHs. Later, we performed a Spearman correlation analysis to assess whether the mean strengths of EC correlated with clinical assessments in SZ patients and performed a receiver operating characteristic (ROC) analysis to evaluate the diagnostic value of EC for distinguishing SZ patients with AVHs from non-AVHs patients. This study is an exploratory study. Recent studies of SZ in effective or causal connectivity found that SZ patients exhibited a deceased or increased connectivity in several SZrelated regions or circuits (Guo et al., 2014; Cui et al., 2015; Li et al., 2017), and the findings were not exactly consistent. We therefore hypothesized that compared with the non-AVHs group, the AVHs group would demonstrate altered EC in the DMN, which would be correlated with the clinical symptoms of SZ patients.

\section{MATERIALS AND METHODS}

\section{Participants}

A total of $48 \mathrm{SZ}$ patients (28 with AVHs and 20 without AVHs) were recruited from the First Affiliated Hospital of Zhejiang University, who fulfilled the diagnostic criteria for SZ mentioned in the Diagnostic and Statistical Manual of Mental Disorders, Fourth Edition (DSM-IV). The Structured Clinical Interview for DSM Disorders (SCID), routine laboratory tests, and physical and neurological examinations were administered for each participant by two clinicians. Inclusion criteria for patients to enter this study were as follows: (1) age between 13 and 45 years; (2) a DSM-IV diagnosis of SZ; (3) first episode of SZ onset; (4) being antipsychotic drug naïve; and (5) of Han nationality. Schizophrenia patients with AVHs required a score of $\geq 3$ on the hallucinatory behavior (P3) item of the Positive and Negative Syndrome Scale (PANSS) (Kay et al., 1987). 

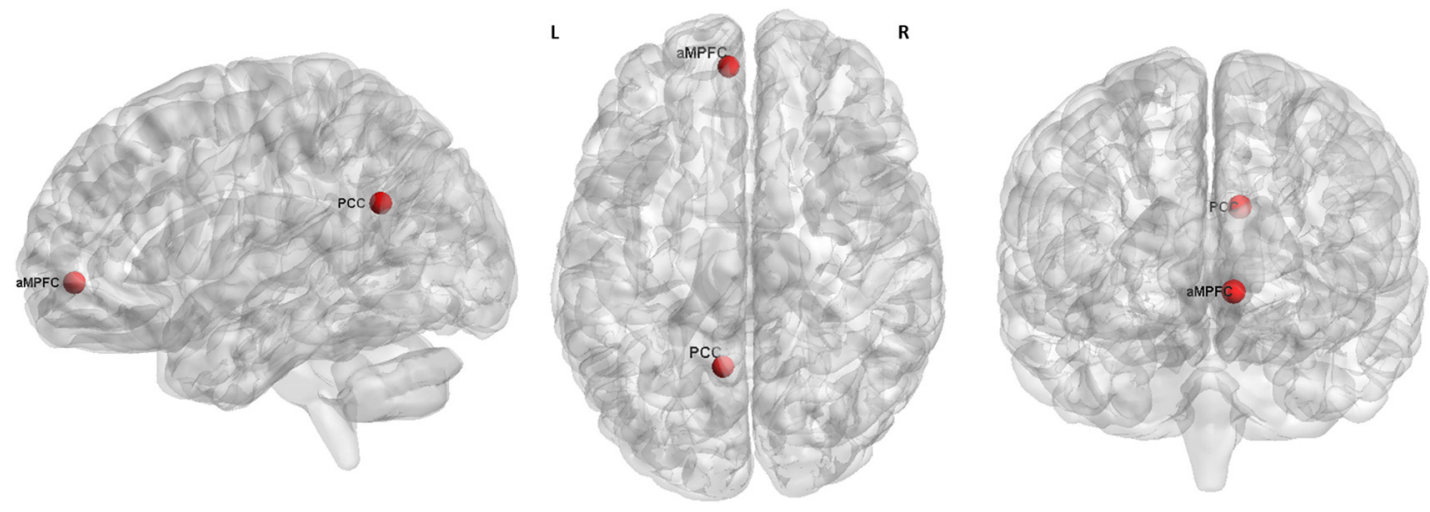

FIGURE 1 | The seed regions (red) for GCA analysis. L, left. R, right. aMPFC, MNI coordinate = $-6,52,-2$, radius $=6 \mathrm{~mm}$. PCC, MNI coordinate = $-8,-56,26$, radius $=6 \mathrm{~mm}$.

The recruited patients included both male $(N=23 ; \mathrm{AVH}=13)$ and female $(N=25 ; \mathrm{AVH}=15)$ participants. A patient was excluded if any of the following conditions was met: (1) a history or presence of any severe unstable systemic disease; (2) a history of brain tumor, cerebral trauma, seizure disorder, mental retardation, or MRI evidence of structural brain abnormalities; (3) being pregnant, lactating, or planning to be pregnant within the following 6 months; and (4) any contraindication or incompatibility for MRI. Clinical symptoms were quantified with PANSS. Moreover, a sample of 38 age-, gender-, handedness-, and education-matched healthy participants were selected to compose a control group. All participants provided written informed consent in accordance with the Declaration of Helsinki. The Institutional Review Board (IRB) protocol was approved by the Research Ethics Committee of the First Affiliated Hospital, College of Medicine, Zhejiang University.

\section{MRI Data Acquisition}

All data were acquired using a Philips Achieva3.0T TX MRI system (Philips Healthcare, Netherlands), which was equipped with an eight-channel head coil array. The rs-fMRI data were acquired along the axial direction in a sequential mode using a fast field echo-echo planar imaging (FFEEPI) sequence. The imaging parameters were as follows: 24 slices, repetition time (TR)/echo time $(\mathrm{TE})=2000 / 35 \mathrm{~ms}$, flip angle $(\mathrm{FA})=80^{\circ}$, slice thickness $/$ gap $=5.0 / 1.0 \mathrm{~mm}$, voxel size $=2.4 \times 2.4 \times 5.0 \mathrm{~mm}^{3}$, matrix $=100 \times 100$, and field of view $(F O V)=240 \times 240 \mathrm{~mm}^{2}$. The rs-fMRI scan lasted for $6 \mathrm{~min}$ and $48 \mathrm{~s}$, and we collected a total of 200 image volumes. Additionally, individual three-dimensional high-resolution T1weighted images were also acquired for the purpose of spatial normalization using a fast field echo sequence: 150 slices, $\mathrm{TR} / \mathrm{TE}=7.5 / 3.7 \mathrm{~ms}$, matrix $=240 \times 240$, slice thickness $=1 \mathrm{~mm}$, $\mathrm{FOV}=240 \times 240 \mathrm{~mm}^{2}$, voxel size $=1 \times 1 \times 1 \mathrm{~mm}^{3}$, and $\mathrm{FA}=8^{\circ}$. There were no obvious structural damages on the conventional MRI scans of each participant as examined by two experienced radiologists.

\section{Data Preprocessing}

Preprocessing of the fMRI data was performed using the Advanced DPARSF ${ }^{1}$ and SPM8 ${ }^{2}$ toolkits. The first 10 functional volumes were discarded to ensure steady state longitudinal magnetization. The remaining 190 volumes were slice-time corrected relative to the middle axial slice to account for the temporal difference in acquisition among the different slices and were then co-registered to correct for head motion during the scan. None of the translation or rotation parameters in any given data set exceeded $2 \mathrm{~mm}$ or $2^{\circ}$. Subsequently, white matter, cerebrospinal fluid, and Friston 24-parameter model of head motion (Friston et al., 1996) were removed as nuisance variables. Functional images were spatially normalized to standard stereotaxic coordinates of the Montreal Neurological Institute (MNI) space and resampled into a voxel size of $3 \mathrm{~mm} \times 3 \mathrm{~mm} \times 3 \mathrm{~mm}$. The data were then smoothed by convolution with an isotropic Gaussian kernel at a full width half maximum (FWHM) of $6 \mathrm{~mm}$ to decrease spatial noise. Finally, we removed linear trends from the time courses and used temporal band-pass filtering $(0.01-0.1 \mathrm{~Hz})$ to remove the effects of low frequency drift and high frequency noise, such as respiratory and heart rhythms.

\section{Definition of Seed Region of Interest (ROI)}

According to the previous reports (Andrews-Hanna et al., 2010; Alonso-Solís et al., 2015), we selected the two hubs of the DMN for resting-state EC analysis, which were defined as a sphere with a radius of $6 \mathrm{~mm}$ and centered at the peak MNI coordinates of $-6,52,-2$ (aMPFC) and $-8,-56,26$ (PCC), respectively (Figure 1).

\section{Effective Connectivity Analysis}

We calculated the EC between the reference time series of the ROI and the time series of each voxel in the whole brain using REST-GCA in the REST toolbox ${ }^{1}$, which is a popular

\footnotetext{
${ }^{1}$ http://www.restfmri.net

${ }^{2}$ http://www.fil.ion.ucl.ac.uk/spm
} 
resting-state fMRI data analysis toolkit (Song et al., 2011; Yu et al., 2014; Zuo and Xing, 2014). In addition to EC analysis, this toolkit also includes some other analytic methods, such as functional connectivity analysis based on linear correlation, regional homogeneity, amplitude of low frequency fluctuation (ALFF), and fractional ALFF [For more details related to GCA, see our previous study (Zhao et al., 2016)]. Finally, the GCA maps for all subjects were converted to $z$-values to improve the normality using Fisher's r-to-z transformation.

\section{Statistical Analysis}

A two-sample $t$-test was used to compare the difference between each pair of all three groups [AlphaSim corrected significance level of $P<0.05$ (combined voxel height threshold of $P<0.005$ and an extent threshold of $k>69$ voxels according a Monte Carlo simulation)] within the gray matter (GM) mask with age, gender, and mean framewise displacement (FD) of Jenkinson (Jenkinson et al., 2002) as covariates. In this study, we primarily focused on the differences in EC in the DMN between AVHs group and non-AVHs group. To examine the correlations between mean EC strength and PANSS scores, a Spearman correlation analysis was performed for each patient group. Additionally, an ROC analysis was performed to assess the diagnostic capability of these imaging measures to distinguish SZ patients with AVHs from those without AVHs. Correlation and ROC analyses were performed using the SPSS software. The threshold of significance was set at $P<0.05$.

\section{Validation Analysis}

We were also interested in evaluating whether our main results were affected by GM atrophy and by using different preprocessing/analysis strategies (including head motion correction and global signal removal). The relevant procedures are described as follows.

\section{The Effects of GM Loss}

Previous studies suggested that functional analysis results could potentially be influenced by structural GM differences among groups (Wang et al., 2011; Dai et al., 2014). To explore the possible confounding effect of GM atrophy, we performed a voxel-based morphometry analysis on structural MRI images and took the GM volume (GMV) (i.e., modulated images) as a covariate in the GCA statistical analyses. Briefly, individual GMV maps in the standard space were obtained by a unified segmentation algorithm as described previously (Ashburner and Friston, 2005). After spatially smoothing with a $10-\mathrm{mm}$ FWHM Gaussian kernel, a two-sample $t$-test was performed with age and gender as covariates. The threshold of statistical significance was set at $P<0.05$ and cluster size $>642$ voxels, which corresponded to an Alphasim corrected $P<0.05$ according to a Monte Carlo simulation within a GM network mask using the dpabi software (Yan et al., 2016). Later, we extracted the averaged GMV in the regions with a significant between-group difference as the covariate to perform the GCA again.

\section{The Effects of Head Motion and Global Signal Head motion}

Recently, evidence showed that head motion has a confounding effect on functional connectivity analysis (Power et al., 2012; Yan et al., 2013). In this study, we failed to find significant differences in head motion between AVHs group and non-AVHs group [twotailed two-sample $t$-test: $P=0.33$ for translational, $P=0.11$ for rotational, $P=0.66$ for mean FD of Jenkinson]. Although the Friston 24-parameter model of head motion was regressed in the preprocessing and the statistical analysis was performed by including mean FD as an additional covariate (Yan et al., 2013), to exclude any possible effects of head motion, we re-performed a 'scrubbing' procedure on the preprocessed images (Power et al., 2012; Yan et al., 2013). For each subject, rs-fMRI volumes were first censored based on a criterion of FD $>0.2 \mathrm{~mm}$, and the GCA analysis was then reanalyzed using these censored rs-fMRI data.

\section{Global signal removal}

Currently, it is still controversial whether global signal should be removed in rs-fMRI preprocessing. Several previous studies have suggested that the global signal is associated with nonneuronal activity such as respiration and should be removed (Birn et al., 2006; Chang and Glover, 2009). However, the removal of the global signal introduces widespread negative functional connectivities (Murphy et al., 2009; Weissenbacher et al., 2009). To explore the effects of global signal removal on our results, we reanalyzed our data regressed on the global signal.

\section{RESULTS}

\section{Characteristics of Participants}

No significant differences were detected among the three groups in terms of age, sex, and education level. In addition, the two patient groups had no significant differences in course of illness, age of first onset, and PANSS scores except for PANSS total scores $(p<0.05$, AVHs $>$ non-AVHs $)$ and PANSS positive scores $(p<0.01$ AVHs $>$ non-AVHs) (Table 1$)$.

TABLE 1 | Demographic and clinical characteristics of patients with AVHs $(n=28)$, patients without AVHs $(n=20)$, and HCs $(n=38)$.

\begin{tabular}{lccc}
\hline Measure & AVHs & Non-AVHs & HCs \\
\hline Age (years) $^{\mathrm{a}}$ & $24.32 \pm 8.46$ & $24.35 \pm 6.94$ & $25.44 \pm 7.52$ \\
Sex(male/female) $^{\mathrm{b}}$ & $13 / 15$ & $10 / 10$ & $17 / 21$ \\
Handedness & $\mathrm{R}$ & $\mathrm{R}$ & $\mathrm{R}$ \\
Education (years) $^{\mathrm{a}}$ & $11.29 \pm 3.00$ & $11.70 \pm 2.60$ & $13.34 \pm 3.58$ \\
Course of illness (months) $^{\mathrm{a}}$ & $17.78 \pm 22.57$ & $18.60 \pm 15.22$ & - \\
Age of first onset (years) $^{\mathrm{a}}$ & $22.75 \pm 7.76$ & $22.85 \pm 7.69$ & - \\
PANSS total scores $^{\mathrm{a}}$ & $88.11 \pm 16.89^{*}$ & $78.60 \pm 12.55$ & - \\
PANSS P scores $^{\mathrm{a}}$ & $23.57 \pm 4.66^{* *}$ & $18.95 \pm 6.37$ & - \\
PANSS N scores $^{\mathrm{a}}$ & $20.54 \pm 8.58$ & $18.5 \pm 7.39$ & - \\
PANSS G scores $^{\mathrm{a}}$ & $39.61 \pm 7.97$ & $37.10 \pm 7.88$ & - \\
PANSS S scores $^{\mathrm{a}}$ & $4.39 \pm 2.06$ & $4.05 \pm 1.73$ & -
\end{tabular}

Data are mean $\pm S D .{ }^{*} p<0.05$ and ${ }^{* *} p<0.01$ AVHs vs. Non-AVHs. PANSS, Positive and Negative Syndrome Scale. ${ }^{a}$ t-test. ${ }^{b} \chi^{2}$ test. 


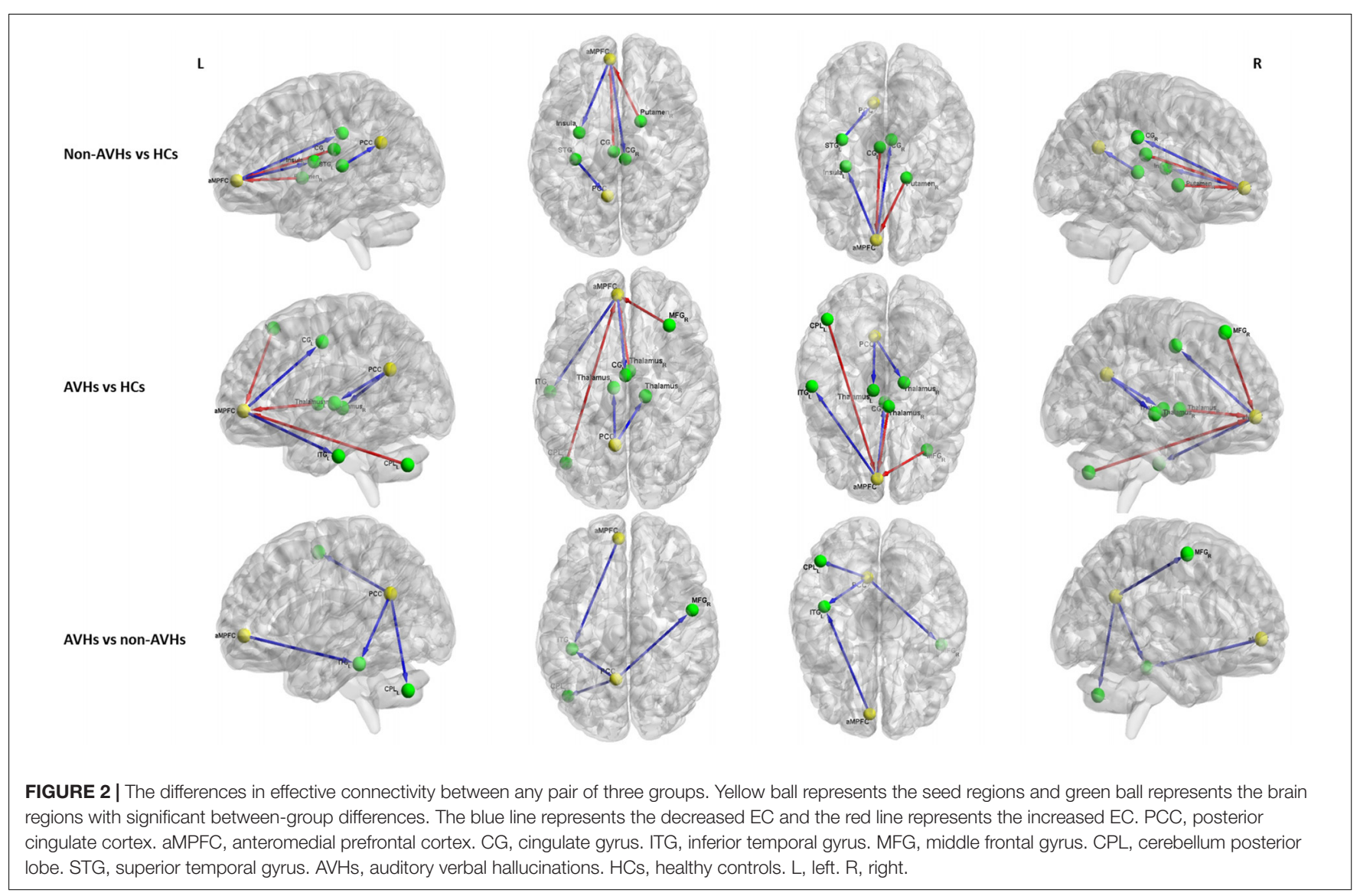

\section{Effective Connectivity}

For the aMPFC, two-sample $t$-tests revealed a significantly lower EC from aMPFC to left inferior temporal gyrus (ITG) in SZ patients with AVHs than those without AVHs. Compared with HCs, the non-AVHs group displayed reduced EC from aMPFC to right cingulate gyrus and left insula and enhanced $\mathrm{EC}$ from left cingulate gyrus and right putamen to aMPFC, while SZ patients with AVHs showed a decreased EC from aMPFC to left cingulate gyrus and left ITG and increased EC from right middle frontal gyrus (MFG), right thalamus, and left cerebellum posterior lobe (CPL) to aMPFC (Figure 2 and Table 2).

For the PCC, relative to those without AVHs, SZ patients with AVHs showed decreased EC from PCC to left CPL, left ITG, and right MFG. Moreover, the non-AVHs group demonstrated reduced EC from left superior temporal gyrus to PCC, and the AVHs group displayed lower EC from PCC to bilateral thalamus when compared with the HCs (Figure 2 and Table 3 ).

\section{Correlation and ROC Analysis}

We extracted the average EC in the four regions that displayed a significant difference between the two patients groups. Later, a Spearman correlation analysis was performed between mean EC and duration of illness as well as PANSS scores across all the patients. Significant negative correlations were observed between the EC from aMPFC to left ITG and the PANSS positive scores $(r=-0.402, p=0.034)$ (Figure 3A) in the
AVHs group. Significant negative correlations, between (1) the EC from aMPFC to left ITG and the PANSS positive scores $(r=-0.571, p=0.009)$ (Figure 3B) and (2) the EC from PCC to left ITG and the PANSS negative scores $(r=-0.610, p=0.004)$ (Figure 3C), were both observed in the non-AVHs group (uncorrected). However, these correlations failed to survive when the multiple comparisons were corrected for $p<0.05$ by FDR, perhaps owing to the limited statistical power provided by the limited size of the samples. Additionally, we displayed the mean strength for each EC with between-group difference (Figure 3D) and used ROC analysis to evaluate the diagnostic value of EC strength (Figure 3E). The area under the ROC for aMPFC-ITG connectivity $(0.83 ; 95 \%$ confidence interval, $0.70-$ $0.96)$, PCC-ITG connectivity $(0.80 ; 95 \%$ confidence interval, $0.67-0.93)$, PCC-MFG connectivity $(0.74 ; 95 \%$ confidence interval, $0.60-0.88)$, and PCC-CPL connectivity $(0.79 ; 95 \%$ confidence interval, $0.65-0.93$ ) suggested that these connections have clinical diagnostic value for distinguishing SZ patients with AVHs from non-AVHs.

\section{Validation Results}

The assessment of the effects of GM atrophy and head motion correction and global signal removal on our main findings between the AVHs group and the non-AVHs group showed the following results. (1) The effects of GM loss: There was no significant difference in GMV between the AVHs group 
TABLE 2 | Regions showing significant differences in effective connectivity in aMPFC between any pairs of three groups.

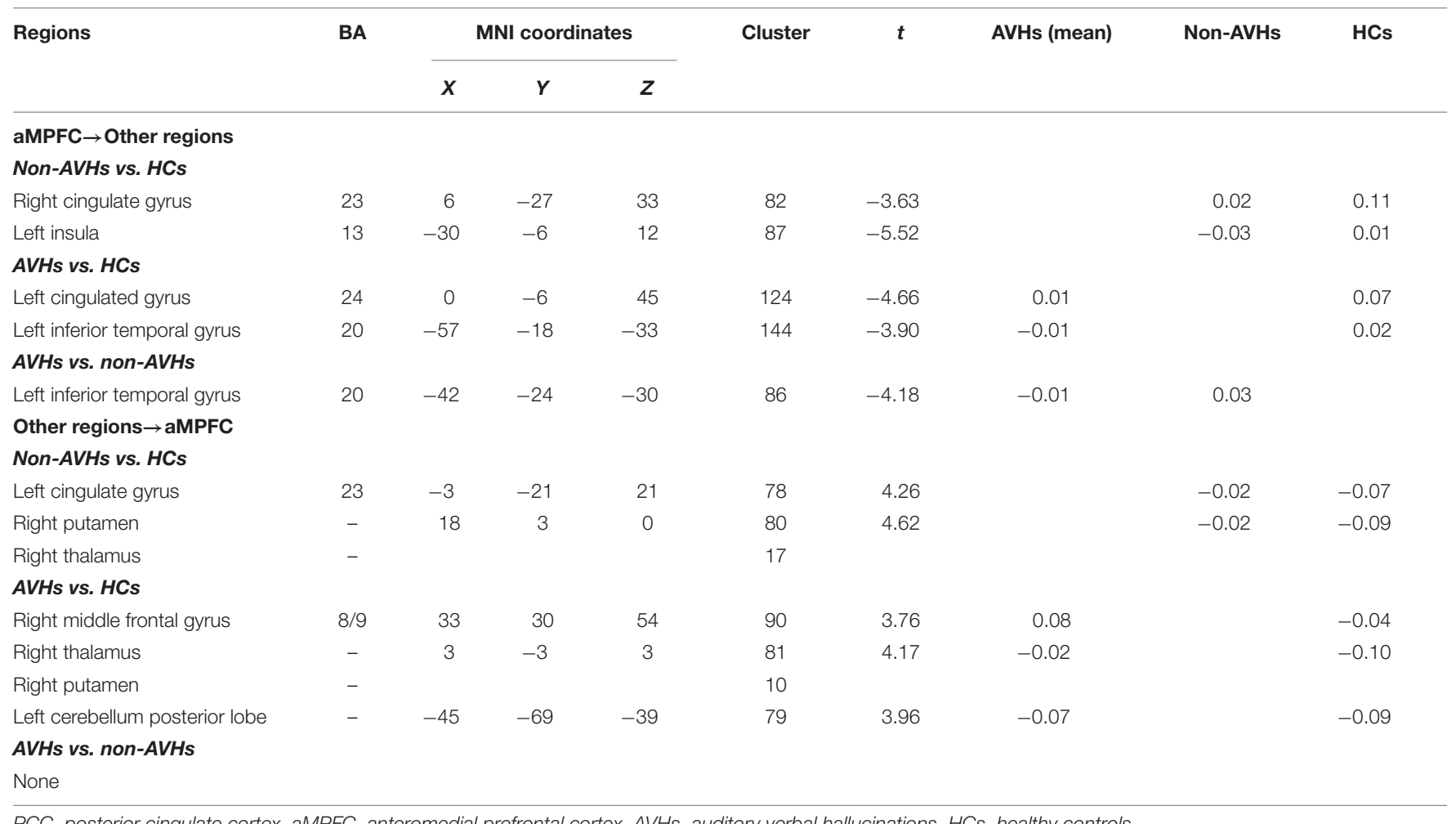

PCC, posterior cingulate cortex. aMPFC, anteromedial prefrontal cortex. AVHs, auditory verbal hallucinations. HCs, healthy controls.

TABLE 3 | Regions showing significant differences in effective connectivity in PCC between any pairs of three groups.

\begin{tabular}{|c|c|c|c|c|c|c|c|c|c|}
\hline \multirow[t]{2}{*}{ Regions } & \multirow[t]{2}{*}{ BA } & \multicolumn{3}{|c|}{ MNI coordinates } & \multirow[t]{2}{*}{ Cluster } & \multirow[t]{2}{*}{$t$} & \multirow[t]{2}{*}{ AVHs (mean) } & \multirow[t]{2}{*}{ Non-AVHs } & \multirow[t]{2}{*}{ HCs } \\
\hline & & $x$ & $Y$ & $z$ & & & & & \\
\hline
\end{tabular}

\section{$\mathrm{PCC} \rightarrow$ Other regions}

Non-AVHs vs. HCs

None

AVHs vs. HCs

Left thalamus

Right thalamus

\section{AVHs vs. non-AVHs}

Right middle frontal gyrus

Left inferior temporal gyrus

Left cerebellum posterior lobe

Other regions $\rightarrow$ PCC

Non-AVHs vs. HCs

Left superior temporal gyrus

AVHs vs. HCs

None

\section{AVHs vs. non-AVHs}

None

PCC, posterior cingulate cortex. aMPFC, anteromedial prefrontal cortex. AVHs, auditory verbal hallucinations. HCs, healthy controls.

and the non-AVHs group, either in local regions or in the whole brain (AVHs: $0.33 \pm 0.06$ (mean \pm SD); non-AVHs: $0.34 \pm 0.04 ; p=0.37$ ). (2) The effects of head motion: Using the statistical analysis accounting for the 'scrubbing' procedure in preprocessed images (Power et al., 2012; Yan et al., 2013), we found that the main results in ECs from aMPFC to ITG and from PCC to both ITG and MFG survived (Alphasim corrected $P<0.05$ ) (Figure 4). (3) The effects of global signal removal: With the global signal removed, the AVHs group showed significantly decreased EC from aMPFC to ITG 

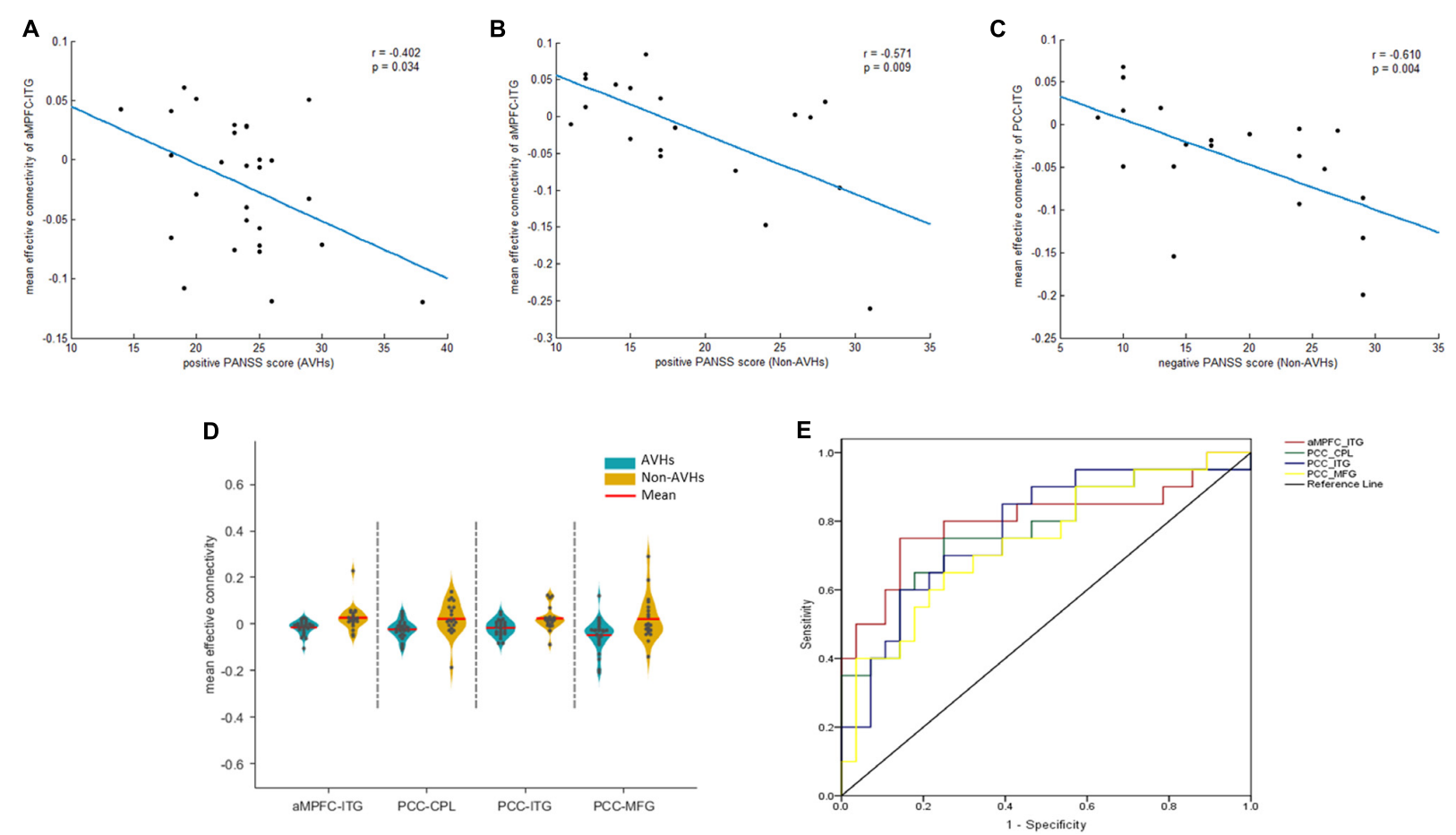

FIGURE 3 | Correlation and ROC analysis results. The significant correlations between mean EC and clinical assessments (A-C). The quantitative display of mean ECs in the four regions with between-groups differences (D) and ROC results (E). PCC, posterior cingulate cortex. aMPFC, anteromedial prefrontal cortex. ITG, inferior temporal gyrus. MFG, middle frontal gyrus. CPL, cerebellum posterior lobe. CPL, cerebellum posterior lobe. AVHs, auditory verbal hallucinations. L, left. R, right.

(Alphasim corrected $P<0.05$ ), which was consistent with our results without removing the global signal. However, the EC from PCC to ITG, MFG, and CPL exhibited non-significant results after global signal removal (Figure 4). It was not solved why the removal of the global signal produced such an effect on EC in this study. This should be further investigated in the future.

\section{DISCUSSION}

In this study, GCA results revealed hypoconnectivity from aMPFC to left ITG and from PCC to left CPL, left ITG, and right MFG in the AVHs group but not in the non-AVHs group. In comparison with HCs, SZ patients with AVHs showed decreased EC from aMPFC to left cingulate gyrus and left ITG and from PCC to bilateral thalamus and increased EC from right MFG, right putamen, and left CPL to aMPFC. The non-AVHs group displayed reduced EC from aMPFC to right cingulate gyrus and left insula and from left superior temporal gyrus to PCC and enhanced EC from left cingulate gyrus, right thalamus, and right putamen to aMPFC relative to HCs. In addition, EC from aMPFC to left ITG was negatively correlated with PANSS positive scores in the two patient groups, and EC from PCC to left ITG was negatively correlated with PANSS negative scores in the nonAVHs group. These findings support our hypothesis and suggest that the information flows of the DMN are relatively disruptive or weaker in SZ patients with AVHs than in those without AVHs, and these changes are correlated with the clinical symptoms of SZ patients. This insight may be helpful to understand the potential neuromechanism underlying AVHs of SZ patients.

Although most rs-fMRI studies have found changes in functional connectivity between DMN and non-DMN brain regions, and also between DMN and other brain networks in SZ patients, the results were somewhat controversial. For example, several studies showed decreased functional connectivity within the DMN in SZ patients compared with HCs (Rotarska-Jagiela et al., 2010; Camchong et al., 2011), while some other studies found increased connectivity within the DMN and between DMN and non-DMN brain regions (Mingoia et al., 2012; Alonso-Solís et al., 2015). Therefore, further studies of functional connectivity are needed to elucidate the role of the DMN in SZ patients. Recently, a spectral DCM study identified reduced EC from the PCC to the anterior frontal node of the DMN in first-episode SZ patients, reflecting the reduced postsynaptic efficacy of prefrontal afferents (Bastos-Leite et al., 2014). Another DCM study in SZ patients found altered EC related to the medial prefrontal cortex, anterior part of the DMN, indicating hippocampaldorsolateral prefrontal-medial prefrontal hypoconnectivity (Cui et al., 2015). Additionally, SZ patients have shown decreased PCC connectivity with other parts of the DMN compared with HCs, which could then lead to a widespread impairment of communication within the DMN (Bluhm et al., 2007; RotarskaJagiela et al., 2010). This study found that, compared with HCs, SZ patients showed significantly altered information flows 


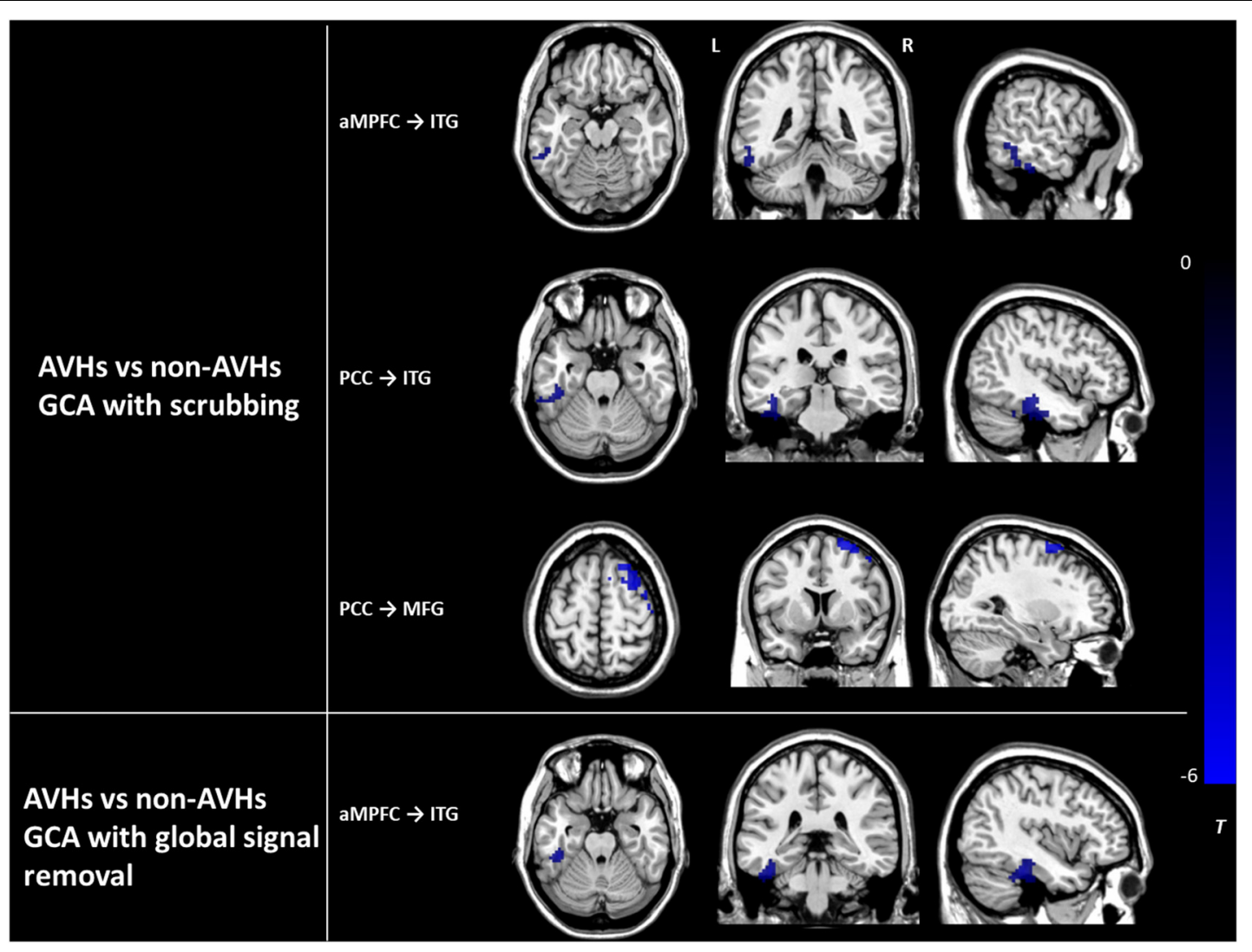

FIGURE 4 | The differences in effective connectivity between the AVHs group and the non-AVHs group with scrubbing and global signal removal, respectively. Color bar represents the $t$-value. PCC, posterior cingulate cortex. aMPFC, anteromedial prefrontal cortex. ITG, inferior temporal gyrus. MFG, middle frontal gyrus. AVHs, auditory verbal hallucinations. L, left. $R$, right.

between the DMN and several SZ-related regions. Specifically, the patient group exhibited decreased efferent information from aMPFC to bilateral cingulate gyrus and from PCC to right MFG and increased afferent information from left cingulate gyrus and right MFG to aMPFC, which were consistent with recent reports on EC of SZ patients (Bastos-Leite et al., 2014; Cui et al., 2015). Additionally, we also found that SZ patients showed abnormal EC between DMN and inferior/superior temporal gyrus, CPL as well as subcortical regions, such as insula, putamen and thalamus, which were not found in the previous studies, but the regions have been consistently reported to be functionally or structurally aberrant in SZ patients in the previous studies (Kuroki et al., 2006; Fornito et al., 2012; Alderson-Day et al., 2015, 2016). Thus, from the perspective of information transmission, these results suggest that the etiology of SZ patients may disrupt the normal processes of DMN network integration and segregation (Karbasforoushan and Woodward, 2012; Alonso-Solís et al., 2015).

With regard to AVH-specific connectivity, our results found that SZ patients with AVHs showed reduced EC from aMPFC to left ITG and from PCC to right MFG, left ITG, and left CPL relative to the non-AVHs group. Similarly, Lawrie et al. (2002) found that the predisposition to hallucinate may be associated with impaired functional connectivity between frontal and temporal brain areas. de la Iglesia-Vaya et al. (2014) uncovered an anomalous process of neural connectivity in the cortico-cerebellar-thalamic-cortical circuits in patients with auditory hallucinations. Additionally, it has been reported that the DMN may be relevant for generating, monitoring, and updating self-referential images, which is possibly based on effective and autobiographical knowledge (Van De Ven, 2013). Early neuropsychological theories have suggested that AVHs might result from a failure in correctly monitoring internally generated speech events (Frith, 2005). A recent review of auditory hallucinations demonstrated that hallucinations were associated with the weaker integrity of the DMN, suggesting that unstable DMN states may be an important precursor to auditory hallucination states (Alderson-Day et al., 2016). Moreover, the interaction of the DMN with other RSNs (Alonso-Solís et al., 2015) and dynamic stability of the DMN are both implicated in hallucinatory states and traits (Jardri et al., 2013). Thus, the aberrant functional architecture of the DMN may contribute to misinterpretations of agency in SZ, which internally generated auditory images or speech and could, in turn, lead to AVHs (Frith, 2005; Wible et al., 2009). Therefore, the findings in this study suggest that the abnormal information flows of the DMN could cause an incorrect monitoring function and interpretation 
of agency processing in SZ patients with AVHs, which could consequently result in the generation of AVHs in patients.

More importantly, we found that the mean EC from aMPFC to left ITG was negatively correlated with the positive PANSS score, and the mean EC from PCC to left ITG was negatively correlated with the negative PANSS score. Therefore, the left ITG intuitively seems to play a crucial role in the pathogenetic mechanism of AVHs for SZ patients. The ITG locates below the middle temporal gyrus and is connected at the back with the inferior occipital gyrus, which is a part of sensorimotor networks involving auditory and language regions (AldersonDay et al., 2016). In early studies, the ITG was demonstrated to be probably involved in visual word grapheme system and semantic processing (Nobre et al., 1994; Binder et al., 2000), and it also plays an important role in both dorsal and ventral visual pathways (Sewards and Sewards, 2002). Subsequent studies revealed smaller GMV of bilateral posterior ITG in first-episode and chronic SZ (Onitsuka et al., 2004; Kuroki et al., 2006). Moreover, the GMV of the left posterior ITG was negatively correlated with the factor score for anxiety/depression, and the smaller GMV in the ITG may be related to the pathology common to SZ and affective psychoses (Kuroki et al., 2006). Available evidence suggest that the primary deficit in SZ may be a failure to attenuate precision at the lowest (sensory) cortical levels, leading to a failure of sensory attenuation and characteristic soft neurological signs (e.g., abnormal pursuit eye movements) (Adams et al., 2013). This primary deficit is assumed to induce compensatory increases in the precision of higher levels and consequent difficulties inferring the causes of sensationscausing, for example, hallucinations and delusions (Brown et al., 2013). Therefore, we speculate that the decreased EC from DMN to left ITG and the relation with clinical assessments in this study provide supportive evidence for the assumption that the changes of information from DMN to left ITG could have an effect on the communication between low- and high-level cortical regions in SZ patients and have resulted in the production of AVHs.

Several limitations in this study should be noted. First, the sample size is limited owing to the strict recruitment criteria, including the requirements of drug-naïve and firstepisode patients. This could be the reason why the correlation significance failed to survive the FDR correction. A larger sample size is needed for improved statistical power and to further confirm our findings. Second, the seed-based approach was required to manually predefine the seed regions. It could be improved using other analytic methods that overcome this flaw, such as independent component analysis (ICA). However, ICA requires the challenging step of defining the number of

\section{REFERENCES}

Adams, R. A., Stephan, K. E., Brown, H. R., Frith, C. D., and Friston, K. J. (2013). The computational anatomy of psychosis. Front. Psychiatry 4:47. doi: 10.3389/ fpsyt.2013.00047

Alderson-Day, B., Diederen, K., Fernyhough, C., Ford, J. M., Horga, G., Margulies, D. S., et al. (2016). Auditory hallucinations and the brain's resting-state components in advance, and using the inappropriate component number in ICA is likely to produce region of interests (ROIs) totally different from what we would like to examine. While every other method bears certain intrinsic shortcomings, we deemed that the seed-based method suited our needs best and, therefore, it was adopted. Finally, the EC analysis in this study only revealed directional functional connectivity. In the future employing a multimodal analysis that exhibits other data modalities, including diffusion tensor imaging, magnetic resonance spectroscopy, electroencephalography, and positron emission tomography, may significantly strengthen the reliability of the findings in this study.

In conclusion, this study has uncovered decreased EC from aMPFC to left ITG and from PCC to left CPL, left ITG, and right MFG in SZ patients with AVHs than those without AVHs using the seed-based GCA approach based on rs-fMRI data. The ROC analysis also shows that the EC in the four brain regions is able to distinguish AVHs patients from non-AVHs patients. More importantly, the mean EC of the left ITG shows significant correlations with clinical assessments in SZ patients. These findings demonstrate that the disrupted information flows of the DMN may cause a failure to internal control on language processing and in turn induced AVHs in SZ patients, and the left ITG plays a vital role in the pathophysiologic mechanism underlying AVHs.

\section{AUTHOR CONTRIBUTIONS}

$\mathrm{DX}, \mathrm{MH}$, and $\mathrm{ZZ}$ designed the study. ZZ and DX wrote the manuscript. $\mathrm{MH}$ and YX collected imaging and clinical data. $\mathrm{XL}, \mathrm{GF}, \mathrm{ZS}, \mathrm{SL}$, and YX supported the analysis, interpretation, and manuscript revision. All authors contributed to and have approved the final manuscript.

\section{FUNDING}

This work was supported in part by a grant sponsored by the China National Key R\&D Program (2017YFC1308500/2017YFC 1308502) and a grant sponsored by the China National Science Foundation (81471734). It is also supported partly by a grant of China National Key R\&D Program (2016YFC1307005), a grant of Basic Public Welfare Research Projects in Zhejiang province (LGF18H090003), a grant of Major Subject of Zhejiang Province (2015C03054), and a grant by the International Cooperative Program of Shanghai Municipal Science and Technology Commission (No. 16550720500).

networks: findings and methodological observations. Schizophr. Bull. 42, 1110-1123. doi: 10.1093/schbul/sbw078

Alderson-Day, B., McCarthy-Jones, S., and Fernyhough, C. (2015). Hearing voices in the resting brain: a review of intrinsic functional connectivity research on auditory verbal hallucinations. Neurosci. Biobehav. Rev. 55, 78-87. doi: 10.1016/j.neubiorev.2015. 04.016 
Allen, P., Modinos, G., Hubl, D., Shields, G., Cachia, A., Jardri, R., et al. (2012). Neuroimaging auditory hallucinations in schizophrenia: from neuroanatomy to neurochemistry and beyond. Schizophr. Bull. 38, 695-703. doi: 10.1093/schbul/ sbs066

Alonso-Solís, A., Vives-Gilabert, Y., Grasa, E., Portella, M. J., Rabella, M., Sauras, R. B., et al. (2015). Resting-state functional connectivity alterations in the default network of schizophrenia patients with persistent auditory verbal hallucinations. Schizophr. Res. 161, 261-268. doi: 10.1016/j.schres.2014.10.047

Andrews-Hanna, J. R., Reidler, J. S., Sepulcre, J., Poulin, R., and Buckner, R. L. (2010). Functional-anatomic fractionation of the brain's default network. Neuron 65, 550-562. doi: 10.1016/j.neuron.2010.02.005

Ashburner, J., and Friston, K. J. (2005). Unified segmentation. Neuroimage 26, 839-851. doi: 10.1016/j.neuroimage.2005.02.018

Bastos-Leite, A. J., Ridgway, G. R., Silveira, C., Norton, A., Reis, S., and Friston, K. J. (2014). Dysconnectivity within the default mode in first-episode schizophrenia: a stochastic dynamic causal modeling study with functional magnetic resonance imaging. Schizophr. Bull. 41, 144-153. doi: 10.1093/schbul/sbu080

Binder, J. R., Frost, J. A., Hammeke, T. A., Bellgowan, P. S., Springer, J. A., Kaufman, J. N., et al. (2000). Human temporal lobe activation by speech and nonspeech sounds. Cereb. Cortex 10, 512-528. doi: 10.1093/cercor/10.5.512

Birn, R. M., Diamond, J. B., Smith, M. A., and Bandettini, P. A. (2006). Separating respiratory-variation-related fluctuations from neuronal-activityrelated fluctuations in fMRI. Neuroimage 31, 1536-1548. doi: 10.1016/j. neuroimage.2006.02.048

Biswal, B., Yetkin, F. Z., Haughton, V. M., and Hyde, J. S. (1995). Functional connectivity in the motor cortex of resting human brain using echo-planar MRI. Magn. Reson. Med. 34, 537-541. doi: 10.1002/mrm.1910340409

Bluhm, R. L., Miller, J., Lanius, R. A., Osuch, E. A., Boksman, K., Neufeld, R., et al. (2007). Spontaneous low-frequency fluctuations in the BOLD signal in schizophrenic patients: anomalies in the default network. Schizophr. Bull. 33, 1004-1012. doi: 10.1093/schbul/sbm052

Brown, H., Adams, R. A., Parees, I., Edwards, M., and Friston, K. (2013). Active inference, sensory attenuation and illusions. Cogn. Process. 14, 411-427. doi: 10.1007/s10339-013-0571-3

Buckner, R. L., Andrews-Hanna, J. R., and Schacter, D. L. (2008). The brain's default network. Ann. N. Y. Acad. Sci. 1124, 1-38. doi: 10.1196/annals.1440.011

Camchong, J., MacDonald, A. W., Bell, C., Mueller, B. A., and Lim, K. O. (2011). Altered functional and anatomical connectivity in schizophrenia. Schizophr. Bull. 37, 640-650. doi: 10.1093/schbul/sbp131

Chang, C., and Glover, G. H. (2009). Effects of model-based physiological noise correction on default mode network anti-correlations and correlations. Neuroimage 47, 1448-1459. doi: 10.1016/j.neuroimage.2009.05.012

Cui, L.-B., Liu, J., Wang, L.-X., Li, C., Xi, Y.-B., Guo, F., et al. (2015). Anterior cingulate cortex-related connectivity in first-episode schizophrenia: a spectral dynamic causal modeling study with functional magnetic resonance imaging. Front. Hum. Neurosci. 9:589. doi: 10.3389/fnhum.2015.00589

Cui, L.-B., Liu, L., Guo, F., Chen, Y.-C., Chen, G., Xi, M., et al. (2017). Disturbed brain activity in resting-state networks of patients with first-episode schizophrenia with auditory verbal hallucinations: a cross-sectional functional MR imaging study. Radiology 283, 810-819. doi: 10.1148/radiol.2016160938

Dai, Z., Yan, C., Li, K., Wang, Z., Wang, J., Cao, M., et al. (2014). Identifying and mapping connectivity patterns of brain network hubs in Alzheimer's disease. Cereb. Cortex 25, 3723-3742. doi: 10.1093/cercor/bhu246

de la Iglesia-Vaya, M., Escartí, M. J., Molina-Mateo, J., Martí-Bonmatí, L., Gadea, M., Castellanos, F. X., et al. (2014). Abnormal synchrony and effective connectivity in patients with schizophrenia and auditory hallucinations. Neuroimage Clin. 6, 171-179. doi: 10.1016/j.nicl.2014.08.027

Fornito, A., Zalesky, A., Pantelis, C., and Bullmore, E. T. (2012). Schizophrenia, neuroimaging and connectomics. Neuroimage 62, 2296-2314. doi: 10.1016/j. neuroimage.2011.12.090

Friston, K. J., Williams, S., Howard, R., Frackowiak, R. S., and Turner, R. (1996). Movement-related effects in fMRI time-series. Magn. Reson. Med. 35, 346-355. doi: $10.1002 / \mathrm{mrm} .1910350312$

Frith, C. (2005). The neural basis of hallucinations and delusions. C. R. Biol. 328, 169-175. doi: 10.1016/j.crvi.2004.10.012

Guo, W., Liu, F., Liu, J., Yu, L., Zhang, J., Zhang, Z., et al. (2014). Abnormal causal connectivity by structural deficits in first-episode, drug-naive schizophrenia at rest. Schizophr. Bull. 41, 57-65. doi: 10.1093/schbul/sbu126
Guo, W., Liu, F., Liu, J., Yu, L., Zhang, J., Zhang, Z., et al. (2015). Abnormal causal connectivity by structural deficits in first-episode, drugnaive schizophrenia at rest. Schizophr. Bull. 41, 57-65. doi: 10.1093/schbul/ sbu126

Hutcheson, N. L., Sreenivasan, K. R., Deshpande, G., Reid, M. A., Hadley, J., White, D. M., et al. (2015). Effective connectivity during episodic memory retrieval in schizophrenia participants before and after antipsychotic medication. Hum. Brain Mapp. 36, 1442-1457. doi: 10.1002/hbm.22714

Jardri, R., Thomas, P., Delmaire, C., Delion, P., and Pins, D. (2013). The neurodynamic organization of modality-dependent hallucinations. Cereb. Cortex 23, 1108-1117. doi: 10.1093/cercor/bhs082

Jenkinson, M., Bannister, P., Brady, M., and Smith, S. (2002). Improved optimization for the robust and accurate linear registration and motion correction of brain images. Neuroimage 17, 825-841. doi: 10.1006/nimg.2002. 1132

Karbasforoushan, H., and Woodward, N. (2012). Resting-state networks in schizophrenia. Curr. Top. Med. Chem. 12, 2404-2414. doi: 10.2174/ 156802612805289863

Kay, S. R., Flszbein, A., and Opfer, L. A. (1987). The positive and negative syndrome scale (PANSS) for schizophrenia. Schizophr. Bull. 13, 261-276. doi: 10.1093/ schbul/13.2.261

Kuroki, N., Shenton, M. E., Salisbury, D. F., Hirayasu, Y., Onitsuka, T., Ersner, H., et al. (2006). Middle and inferior temporal gyrus gray matter volume abnormalities in first-episode schizophrenia: an MRI study. Am. J. Psychiatry 163, 2103-2110. doi: 10.1176/ajp.2006.163.12.2103

Lawrie, S. M., Buechel, C., Whalley, H. C., Frith, C. D., Friston, K. J., and Johnstone, E. C. (2002). Reduced frontotemporal functional connectivity in schizophrenia associated with auditory hallucinations. Biol. Psychiatry 51, 1008-1011. doi: 10.1016/S0006-3223(02)01316-1

Li, B., Cui, L.-B., Xi, Y.-B., Friston, K. J., Guo, F., Wang, H.-N., et al. (2017). Abnormal effective connectivity in the brain is involved in auditory verbal hallucinations in schizophrenia. Neurosci. Bull. 33, 281-291. doi: 10.1007/ s12264-017-0101-x

Liao, W., Ding, J., Marinazzo, D., Xu, Q., Wang, Z., Yuan, C., et al. (2011). Small-world directed networks in the human brain: multivariate Granger causality analysis of resting-state fMRI. Neuroimage 54, 2683-2694. doi: 10. 1016/j.neuroimage.2010.11.007

Menon, V. (2011). Large-scale brain networks and psychopathology: a unifying triple network model. Trends Cogn. Sci. 15, 483-506. doi: 10.1016/j.tics.2011. 08.003

Mingoia, G., Wagner, G., Langbein, K., Maitra, R., Smesny, S., Dietzek, M., et al. (2012). Default mode network activity in schizophrenia studied at resting state using probabilistic ICA. Schizophr. Res. 138, 143-149. doi: 10.1016/j.schres. 2012.01.036

Moran, L. V., Tagamets, M. A., Sampath, H., O’Donnell, A., Stein, E. A., Kochunov, P., et al. (2013). Disruption of anterior insula modulation of largescale brain networks in schizophrenia. Biol. Psychiatry 74, 467-474. doi: 10. 1016/j.biopsych.2013.02.029

Murphy, K., Birn, R. M., Handwerker, D. A., Jones, T. B., and Bandettini, P. A. (2009). The impact of global signal regression on resting state correlations: are anti-correlated networks introduced? Neuroimage 44, 893-905. doi: 10.1016/j. neuroimage.2008.09.036

Nobre, A. C., Allison, T., and McCarthy, G. (1994). Word recognition in the human inferior temporal lobe. Nature 372, 260-263. doi: 10.1038/372260a0

Onitsuka, T., Shenton, M. E., Salisbury, D. F., Dickey, C. C., Kasai, K., Toner, S. K., et al. (2004). Middle and inferior temporal gyrus gray matter volume abnormalities in chronic schizophrenia: an MRI study. Am. J. Psychiatry 161, 1603-1611. doi: 10.1176/appi.ajp.161.9.1603

Palaniyappan, L., Simmonite, M., White, T. P., Liddle, E. B., and Liddle, P. F. (2013). Neural primacy of the salience processing system in schizophrenia. Neuron 79 , 814-828. doi: 10.1016/j.neuron.2013.06.027

Power, J. D., Barnes, K. A., Snyder, A. Z., Schlaggar, B. L., and Petersen, S. E. (2012). Spurious but systematic correlations in functional connectivity MRI networks arise from subject motion. Neuroimage 59, 2142-2154. doi: 10.1016/j. neuroimage.2011.10.018

Roebroeck, A., Formisano, E., and Goebel, R. (2005). Mapping directed influence over the brain using Granger causality and fMRI. Neuroimage 25, 230-242. doi: 10.1016/j.neuroimage.2004.11.017 
Rotarska-Jagiela, A., van de Ven, V., Oertel-Knöchel, V., Uhlhaas, P. J., Vogeley, K., and Linden, D. E. (2010). Resting-state functional network correlates of psychotic symptoms in schizophrenia. Schizophr. Res. 117, 21-30. doi: 10.1016/ j.schres.2010.01.001

Sathian, K., Lacey, S., Stilla, R., Gibson, G. O., Deshpande, G., Hu, X., et al. (2011). Dual pathways for haptic and visual perception of spatial and texture information. Neuroimage 57, 462-475. doi: 10.1016/j.neuroimage.2011.05.001

Schlösser, R., Gesierich, T., Kaufmann, B., Vucurevic, G., Hunsche, S., Gawehn, J., et al. (2003). Altered effective connectivity during working memory performance in schizophrenia: a study with fMRI and structural equation modeling. Neuroimage 19, 751-763. doi: 10.1016/S1053-8119(03)00106-X

Sewards, T. V., and Sewards, M. A. (2002). The medial pain system: neural representations of the motivational aspect of pain. Brain Res. Bull. 59, 163-180. doi: 10.1016/S0361-9230(02)00864-X

Song, X.-W., Dong, Z.-Y., Long, X.-Y., Li, S.-F., Zuo, X.-N., Zhu, C.-Z., et al. (2011). REST: a toolkit for resting-state functional magnetic resonance imaging data processing. PLoS One 6:e25031. doi: 10.1371/journal.pone.0025031

Van De Ven, V. (2013). "Brain functioning when the voices are silent: aberrant default modes in auditory verbal hallucinations," in The Neuroscience of Hallucinations, eds R. Jardri, A. Cachia, P. Thomas, and D. Pins (New York, NY: Springer), 393-415.

Vercammen, A., Knegtering, H., den Boer, J. A., Liemburg, E. J., and Aleman, A. (2010). Auditory hallucinations in schizophrenia are associated with reduced functional connectivity of the temporo-parietal area. Biol. Psychiatry 67, 912918. doi: 10.1016/j.biopsych.2009.11.017

Wang, Z., Yan, C., Zhao, C., Qi, Z., Zhou, W., Lu, J., et al. (2011). Spatial patterns of intrinsic brain activity in mild cognitive impairment and alzheimer's disease: a resting-state functional MRI study. Hum. Brain Mapp. 32, 1720-1740. doi: 10.1002/hbm. 21140

Weissenbacher, A., Kasess, C., Gerstl, F., Lanzenberger, R., Moser, E., and Windischberger, C. (2009). Correlations and anticorrelations in resting-state functional connectivity MRI: a quantitative comparison of preprocessing strategies. Neuroimage 47, 1408-1416. doi: 10.1016/j.neuroimage.2009.05.005

Wible, C. G., Preus, A. P., and Hashimoto, R. (2009). A cognitive neuroscience view of schizophrenic symptoms: abnormal activation of a system for social perception and communication. Brain Imaging Behav. 3, 85-110. doi: 10.1007/ s11682-008-9052-1

Wolf, N. D., Sambataro, F., Vasic, N., Frasch, K., Schmid, M., SchönfeldtLecuona, C., et al. (2011). Dysconnectivity of multiple resting-state networks in patients with schizophrenia who have persistent auditory verbal hallucinations. J. Psychiatry Neurosci. 36, 366-374. doi: 10.1503/jpn.110008

Woodward, N. D., Rogers, B., and Heckers, S. (2011). Functional resting-state networks are differentially affected in schizophrenia. Schizophr. Res. 130, 86-93. doi: 10.1016/j.schres.2011.03.010

Yan, C.-G., Cheung, B., Kelly, C., Colcombe, S., Craddock, R. C., Di Martino, A., et al. (2013). A comprehensive assessment of regional variation in the impact of head micromovements on functional connectomics. Neuroimage 76, 183-201. doi: 10.1016/j.neuroimage.2013.03.004

Yan, C.-G., Wang, X.-D., Zuo, X.-N., and Zang, Y.-F. (2016). DPABI: data processing \& analysis for (resting-state) brain imaging. Neuroinformatics 14, 339-351. doi: 10.1007/s12021-016-9299-4

Yu, R., Chien, Y. L., Wang, H. L. S., Liu, C. M., Liu, C. C., Hwang, T. J., et al. (2014). Frequency-specific alternations in the amplitude of low-frequency fluctuations in schizophrenia. Hum. Brain Mapp. 35, 627-637. doi: 10.1002/hbm.22203

Zhao, Z., Huang, T., Tang, C., Ni, K., Pan, X., Yan, C., et al. (2017). Altered restingstate intra-and inter-network functional connectivity in patients with persistent somatoform pain disorder. PLoS One 12:e0176494. doi: 10.1371/journal.pone. 0176494

Zhao, Z., Wang, X., Fan, M., Yin, D., Sun, L., Jia, J., et al. (2016). Altered effective connectivity of the primary motor cortex in stroke: a resting-state fMRI study with granger causality analysis. PLoS One 11:e0166210. doi: 10.1371/journal. pone.0166210

Zuo, X. N., and Xing, X. X. (2014). Test-retest reliabilities of resting-state FMRI measurements in human brain functional connectomics: a systems neuroscience perspective. Neurosci. Biobehav. Rev. 45, 100-118. doi: 10.1016/ j.neubiorev.2014.05.009

Conflict of Interest Statement: The authors declare that the research was conducted in the absence of any commercial or financial relationships that could be construed as a potential conflict of interest.

Copyright (c) 2018 Zhao, Li, Feng, Shen, Li, Xu, Huang and Xu. This is an open-access article distributed under the terms of the Creative Commons Attribution License (CC BY). The use, distribution or reproduction in other forums is permitted, provided the original author(s) and the copyright owner(s) are credited and that the original publication in this journal is cited, in accordance with accepted academic practice. No use, distribution or reproduction is permitted which does not comply with these terms. 